INPLASY

PROTOCOL

To cite: Cao et al. Comparative efficacy of new antidiabetic drugs on cardiovascular and renal outcomes in patients with diabetic kidney disease: A network meta-analysis of cardiovascular and renal outcome trials. Inplasy protocol 2021120070. doi:

10.37766/inplasy2021.12.0070

Received: 15 December 2021

Published: 15 December 2021

Corresponding author:

Qiuhe Ji

jqiuhe@fmmu.edu.cn

Author Affiliation:

Department of Endocrinology and Metabolism, Xijing Hospital, The Air Force Military Medical University, Shaanxi, Xi'an, 710032, China.

Support: AstraZeneca China (Med writing).

Review Stage at time of this submission: Data analysis.

Conflicts of interest:

None declared.

\section{Comparative efficacy of new antidiabetic drugs on cardiovascular and renal outcomes in patients with diabetic kidney disease: A network meta-analysis of cardiovascular and renal outcome trials}

Cao, $\mathrm{H}^{1}$; Liu, T2; Wang, L3; Ji, Q4

Review question / Objective: Among the new antidiabetic drugs, sodium-glucose cotransporter-2 (SGLT-2) inhibitors have been shown to reduce the risk of cardiorenal outcomes in patients with type 2 diabetes and kidney disease (DKD) in renal outcome trials. Data is also available from CVOTs for the efficacy of glucagon-like peptide 1 receptor agonists (GLP-1 RAs) and dipeptidyl peptidase-4 inhibitors in patients with DKD. However, the relative efficacy of these three drug classes for the prevention of cardiorenal outcomes in patients with DKD is unclear.

Condition being studied: This systematic review and metaanalysis will evaluate the comparative efficacy of SGLT-2 inhibitors, GLP-1 RAs and DPP4-inhibitors on cardiovascular and renal outcomes in patients with DKD.

INPLASY registration number: This protocol was registered with the International Platform of Registered Systematic Review and Meta-Analysis Protocols (INPLASY) on 15 December 2021 and was last updated on 22 December 2021 (registration number INPLASY2021120070).

\section{INTRODUCTION}

Review question / Objective: Among the new antidiabetic drugs, sodium-glucose cotransporter-2 (SGLT-2) inhibitors have been shown to reduce the risk of cardiorenal outcomes in patients with type 2 diabetes and kidney disease (DKD) in renal outcome trials. Data is also available from CVOTs for the efficacy of glucagonlike peptide 1 receptor agonists (GLP-1 RAs) and dipeptidyl peptidase-4 inhibitors 
in patients with DKD. However, the relative efficacy of these three drug classes for the prevention of cardiorenal outcomes in patients with DKD is unclear.

Condition being studied: This systematic review and meta-analysis will evaluate the comparative efficacy of SGLT-2 inhibitors, GLP-1 RAs and DPP4-inhibitors on cardiovascular and renal outcomes in patients with DKD.

\section{METHODS}

Search strategy: A systematic search of the MEDLINE database (via PubMed) will be conducted using a pre-defined search strategy to identify relevant randomized controlled trials reported in English up to July 2021. The following search algorithm will be used: ("sodium-glucose transporter 2 inhibitors" OR "dipeptidyl-peptidase IV inhibitors" OR "glucagon-like peptide 1") AND ("diabetic nephropathies"[Mesh]) OR "chronic kidney disease") AND ("diabetes mellitus, type 2"[Mesh]) AND (randomized controlled). Each search string will also contain the synonyms and related keywords of the search terms mentioned above.

Participant or population: Adults with type 2 diabetes mellitus and kidney disease.

Intervention: Any SGLT-2 inhibitor or GLP-1 RA or DPP4 inhibitor.

\section{Comparator: Placebo or active controlled.}

Study designs to be included: Prospective, randomized controlled event-driven cardiovascular or kidney outcome trials (Phase III or IV)

Eligibility criteria: Randomized controlled trials assessing the efficacy of SLGT-2 inhibitors, GLP-1 RAs and DPP4 inhibitors on cardiovascular and renal outcomes in patients with DKD.

Information sources: A systematic search of the MEDLINE database (via PubMed) will be conducted using a pre-defined search strategy to identify relevant randomized controlled trials

Main outcome(s): Cardiovascular outcomes: 1. Hospitalization for heart failure; 2. Major adverse cardiovascular events (MACE, defined as a composite of cardiovascular death, nonfatal myocardial infarction, or nonfatal stroke); 3 . Cardiovascular death Kidney outcomes: 1. Kidney-specific composite outcome (defined as doubling of serum creatinine or $4 z 0 \%$ or greater decline in eGFR, development of end-stage kidney disease, or death due to kidney disease); 2. Kidney disease progression outcome (defined as doubling of serum creatinine or $40 \%$ or greater decline in eGFR, development of end-stage kidney disease, death due to kidney disease or death due to cardiovascular disease).

Data management: The studies retrieved via database search will be assessed for relevance according to the titles and abstracts, and then those studies potentially eligible to be included will be assessed for final eligibility according to the inclusion and exclusion criteria. Prespecified data will be extracted from the studies using a standardized Excel data extraction sheet. The pre-specified data items to be extracted include study design, intervention characteristics, baseline characteristics of interest, study outcomes.

Quality assessment / Risk of bias analysis: Two researchers will independently assess risk of bias for included trials using the Cochrane Risk of Bias tool. Any disagreements in the process of risk of bias assessment will be resolved through consensus.

Strategy of data synthesis: The study-level data on number of patients with events, hazard ratios and $95 \%$ confidence intervals for each study will be used to assess the effect sizes. Statistical tests will be performed using STATA 15.0 and the statistical package "netmeta" for network meta-analysis in R (version 4.1.0). All tests will be two-sided and $p<0.05$ will be considered statistically significant. The net 
split results will be presented as forest plots. The "netmeta" package on $R$ will be used to evaluate the consistency between direct and indirect estimates. Potential publication bias will be estimated using funnel plots and Egger's tests.

Subgroup analysis: No subgroup analysis is planned.

Sensitivity analysis: A Bayesian network meta-analysis will be employed as the sensitivity analysis, using the "gemtc" package in $R$ (version 4.1.0).

Language: English.

Country(ies) involved: China.

Keywords: Diabetic Kidney Disease; network meta-analysis; Heart failure; Cardiovascular; Type 2 diabetes; New Antidiabetic Agents; GLP-1 RAs, SGLT-2 inhibitors, DPP4 inhibitors.

Contributions of each author:

Author 1 - Hongwei Cao.

Email: 492223795@qq.com

Author 2 - Tao Liu.

Email: neifenmi@163.com

Author 3 - Li Wang

Email: 1240203886@qq.com

Author 4 - Qiuhe Ji.

Email: jqiuhe@fmmu.edu.cn 